\title{
The influence of liposomal formulation on the incorporation and retention of PNA oligomers
}

Paola Ringhieri, ${ }^{1,2}$ Concetta Avitabile ${ }^{3}$ Michele Saviano, ${ }^{4}$ Giancarlo Morelli, ${ }^{1,2}$ Alessandra Romanelli, ${ }^{1,2, *}$ Antonella Accardo ${ }^{1,2, *}$

${ }^{1}$ Department of Pharmacy, University of Naples “Federico II", via Mezzocannone 16, 80134 Napoli (Italy)

${ }^{2}$ Interuniversity Research Centre on Bioactive Peptides (CIRPeB), via Mezzocannone 16, 80134 Napoli (Italy)

${ }^{3}$ Institute of Biostructure and Bioimaging (IBB), CNR, via Mezzocannone 16, 80134 Napoli (Italy)

${ }^{4}$ Institute of Crystallography (IC), CNR, Via Amendola 122, 70126 Bari (Italy)

*Corresponding authors e-mails: antonella.accardo@unina.it alessandra.romanelli@unina.it

\begin{abstract}
Liposomal formulations composed of phospholipids with different unsaturation degrees, head groups and at different cholesterol content have been tested for the encapsulation of Peptide Nucleic Acid (PNA) oligomers. The best loading capability $(177 \mu \mathrm{g}, \mathrm{ER} \%=87.2)$ was obtained for pure liposomes of phosphatidylglycerol (DOPG) with negatively charged head group. The insertion of a $10-20 \%$ of cholesterol in DOPG based liposomes provides a slight decrease $(\sim 160 \mu \mathrm{g})$ of the PNA loading. On the other hand, the cholesterol addition (20-30\%) slows down the PNA's release ( $27 \%$ ) in fetal bovine serum from the liposomal formulation. Based on the encapsulation and the release properties, PEGylated DOPG liposomes with a percentage of cholesterol of $10-20 \%$ are the optimal formulation for the loading of PNA-a210.
\end{abstract}

Keywords: Peptide Nucleic Acid, liposome, formulation, cholesterol, DOPG.

\section{Introduction}

Oligonucleotide based molecules represent a potent tool to unravel and modulate the expression and the function of genes. In this context the application of oligonucleotide analogues, such as Peptide Nucleic Acids (PNAs), showing resistance to degradation in vivo, high affinity and specificity of binding toward complementary DNA and RNA, is widespread (1-3). Peptide Nucleic Acids, containing a pseudo-peptide backbone in place of the sugar-phosphate backbone, have been tested as antisense, antigene molecules and recently as inhibitors of the function of miRNAs (4-7). The limited efficiency of transfection of Peptide Nucleic Acids in cells severely hampers their application in vivo. In the last few years the challenge has been tackled in many different ways(8). A recently proposed strategy entails the conjugation of PNAs to polyanionic molecules, such as polyaspartic acid peptides, followed by the packing with polycations, including polyethylenimine (PEI) and lipofectamine (5). Extension of a PNA sequence by a 6-mer PNA polyA allows its delivery upon hybridization to a polythymidilic acid containing both N,N-dimethylaminopropyl and octyl thiophosphate triester elements (9). Alternatively, PNAs have been hybridized to DNA and immobilized on cationic nanoparticles (10). PNAs have been also conjugated to a variety of carriers, including peptides, PEI, cholic acid, cholesterol (7; 11-13). A very laborious approach consists in the modification of the PNA backbone by insertion of positively or negatively charged pendant groups at the alpha or gamma position (14-16). In few cases naked PNAs have been delivered to cells: biodegradable poly(lactic-co-glycolic acid) (PLGA) nanoparticles have been used to deliver a triplex forming PNA to CD34 cells (17). Nanoparticles based on PLGA/ polyaminobeta ester derivatized on the surface with the MPG peptide were demonstrated to deliver mixtures of PNA and DNA to CF mouse model and to cause correction on the CFTR gene. Recently, we have 
reported the delivery of a PNA oligomer (PNA-a210) to K562 cells by a liposomal formulation based on egg phosphatidylcholine/ cholesterol/ 1,2-distearoyl-sn-glycero-3-phosphoethanolamine$\mathrm{N}$-[carbonyl-methoxy(polyethylene glycol)2000 (egg PC/CHO/DSPE-PEG2000 at 47/47/6 molar ratio) and we have demonstrated that this formulation triggers low or no toxicity to cells (18). The PNA oligomer targets the noncoding RNA miRNA-210, which is involved in the regulation of raptor and $\gamma$-globin genes and in the erythroid differentiation of leukemic K562 cells(19). Effective downregulation of the miR-210 was observed upon treatment of K562 cells with the liposome encapsulated PNA-a210. Similar formulations, SPC/CHO/DSPE-PEG2000 (47/47/6 molar ratio) and HSPC/CHO/DSPE-PEG2000 (56/39/5) have been reported for the encapsulation of peptides and for the liposomal drug Doxil (20). Replacement of SPC or HSPC by egg PC, which has a higher degree of saturated chains, is expected to improve the stability of the liposomes, essential for the in vivo application of liposomal drugs. Goal of this work is to obtain a liposomal formulation with a higher PNA loading ability, as compared to the previously reported one (18). The toxicity effects, likely due to lipids, will therefore be further reduced. To this aim, we tested a number of liposomal formulations, prepared using phospholipids containing two alkyl chains at eighteen carbon atoms (see Fig. 1). The effects of the unsaturation of the hydrophobic portion, of the head group and of the percentage of the cholesterol were evaluated in terms of structural features, PNA loading and release capabilities of the liposomes.

\section{Materials and methods}

Egg Phosphatidylcholine (Egg PC) 1,2-dioleoyl-sn-glycero-3-phosphocholine (DOPC), 1,2dioleoyl-sn-glycero-3-phosphocholine (DOPG), L- $\alpha$-phosphatidylcholine hydrogenated soy (HSPC), cholesterol (CHO) and 1,2-distearoyl-sn-glycero-3-phosphoethanolamine-N-[carbonylmethoxy(polyethylene glycol)2000] (ammonium salt) (DSPE-PEG2000) were purchased from Avanti Polar Lipids (Alabaster, AL). Dialysis tubing 8-10 kDa MWCO was purchased by Pierce Protein (USA). The PNA-a210 was prepared as described elsewhere (18). The sequence of PNAa210 follows: $\mathrm{H}$-ccgctgtcacacgcacag- $\mathrm{NH}_{2}$.

\subsection{Liposome preparation and PNA loading}

A stock solution of PNA-a210 was prepared in $10 \mathrm{mM}$ phosphate buffer at $\mathrm{pH} 7.4$, containing the $0.2 \%$ wt glycerol. Concentration was determined by UV-Vis measurements, carried out on a Thermo Fisher Scientific Inc (Wilmington, Delaware USA) Nanodrop 2000c spectrophotometer equipped with a $1.0 \mathrm{~cm}$ quartz cuvette (Hellma) using a molar absorptivity $(\varepsilon)$ of $172400 \mathrm{M}^{-1} \mathrm{~cm}^{-1}$ at $\lambda=260 \mathrm{~nm}$. All liposomal formulations, reported in Table 1, were prepared combining the thin film/sonication and the freeze/thawing methods. Briefly, the required amounts of phospholipids and cholesterol were dissolved in $3 \mathrm{~mL}$ of chloroform and the organic solvent was removed under a stream of $\mathrm{N}_{2}$ to obtain a homogeneous film on the wall of the vial. Any trace solvent was further removed keeping the vial under vacuum for 15 minutes. Each dry lipid film was hydrated in $1.0 \mathrm{~mL}$ of $10 \mathrm{mM}$ phosphate buffer and sonicated for 10 minutes. The total lipid concentration was $15 \mathrm{mM}$. Then, $500 \mu \mathrm{L}$ of the PNA-a2 10 stock solution $(1.0 \mathrm{mg} / \mathrm{mL})$ were added to the preformed liposomes; the resulting mixture was frozen/thawed by dipping the glass tube for 1 minute into a dry ice bath for rapid cooling and then transferred into a bath at $65{ }^{\circ} \mathrm{C}$, allowing each sample to thaw out for 4 min, and finally vortexed for another minute. Five cycles were performed to promote the entry of PNAs into the liposomes. The liposomal suspension was extruded 10 times, using a thermobarrel extruder system (Northern Lipids Inc, Vancouver, BC, Canada) under nitrogen through a polycarbonate membrane (Nucleopore Track Membrane $25 \mathrm{~mm}$, Whatman, Brentford, UK) with $0.1 \mu \mathrm{m}$ pore size. Extrusion was performed at different temperatures: $65^{\circ} \mathrm{C}$ for HSPC based liposomes (HSPC-94, HSPC-84, HSPC-74, HSPC-64 and HSPC-47), $25^{\circ} \mathrm{C}$ for all DOPC and DOPG liposomes (DOPC-94, DOPC-84, DOPC-74, DOPC-64, DOPC-47, DOPG-94, DOPG-84, DOPG-74, DOPG-64 and DOPG-47) and for two PC formulation (PC-94 and PC-84); finally 
extrusion at $55^{\circ} \mathrm{C}$ was carried out for PC-74, PC-64 and PC-47. Separation of the liposomal suspension from unencapsulated PNA-a210 was achieved using a Sephadex G50 gel filtration column, pre-equilibrated with phosphate buffer. The amount of unloaded PNA eluted from the column was spectroscopically determined by UV-Vis measurements at $\lambda=260 \mathrm{~nm}$. The encapsulation efficiency (EE\%, defined as the weight ratio of encapsulated PNA versus the amphiphilic molecules forming liposomes) and the encapsulation ratio (ER\%, defined as the weight percentage of PNA encapsulated in the liposomes on the total PNA previously added during preparation) were quantified by subtraction of the amount of removed PNA from the total amount of loaded PNA.

\subsection{DSPE-PEG2000 post-insertion}

Dry DSPE-PEG2000 (an amount corresponding to the $6 \%$ mol of the final formulation) was hydrated for $2 \mathrm{~h}$ with PNA containing liposomes at room temperature under stirring.

\subsection{DLS measurements and serum stability}

Mean diameters, diffusion coefficients $(D)$, polydispersity indexes (P.I.) and Z potentials $(\zeta)$ of filled and empty liposomes were measured by Dynamic Light Scattering technique (DLS). DLS measurements were carried out using a Zetasizer Nano ZS (Malvern Instruments, Westborough, MA) that employs a $173^{\circ}$ backscatter detector. Other instrumental settings are: measurement position (mm): 4.65; attenuator: 8 ; temperature: $25^{\circ} \mathrm{C}$; cell: disposable sizing cuvette. Size measurements were carried out on samples previously prepared, after centrifugation at room temperature at $13,000 \mathrm{rpm}$ for 5 minutes at $2.0 \cdot 10^{-4} \mathrm{M}$ final lipids concentration. For each formulation, both hydrodynamic radii, polydispersity indexes and $\mathrm{Z}$ potentials were calculated as the mean of at least three measurements on three different batches.

For the serum stability measurements, liposomes $(5 \mathrm{mM})$ filled with PNA-a210, prepared as above described, were diluted at $2.5 \mathrm{mM}$ with fetal bovine serum (FBS; Biowest, Nuaillé, France). The colloidal suspension was stirred at $37^{\circ} \mathrm{C}$ for $72 \mathrm{~h}$. Samples withdrawn at different time points (30 min, 1h, 2h, 4h, 8h, 16h, 24h, 28h, 36h, 48h and 72h) were diluted with bi-distilled water (1/50 v/v) and analyzed by dynamic light scattering according to the experimental setting described earlier.

\subsection{PNA-a210 liposomal release}

$1.0 \mathrm{~mL}$ of PNA encapsulated liposomes was placed into a dialysis tubing (8-10 KDa MWCO) sealed at both ends with a knot. The dialysis bag was suspended in a release volume of $15 \mathrm{~mL}$ of 10 $\mathrm{mM}$ phosphate buffer $\mathrm{pH} 7.4$ and incubated with stirring at $37^{\circ} \mathrm{C}$ for $72 \mathrm{~h}$. Before starting with the experiment, we checked that the crossing of the PNA molecules through the dialysis membrane occurred quickly, and that the overall release of PNA from the liposomes to the dialysis bag medium is the rate determining step process. Total PNA-a210 concentration in the dialysis tube was monitored versus time by collecting $2.0 \mathrm{~mL}$ aliquots at different time points from the release medium, and analyzing them via UV/Vis at $260 \mathrm{~nm}$. The same volume of fresh buffer was added immediately to maintain a constant volume in the accepting solution. The extent of PNA-a210 released was evaluated as percentage of the ratio between the amount of released PNA and of the total PNA previously loaded into the liposomes.

\section{Results and Discussion}

Several features of the commercial phospholipids, such as the length, the unsaturation degree of the alkyl portion and the charge of the head-group deeply influence lipid bilayer microfluidity, that is directly responsible for incorporation and retention of pharmaceutically relevant molecules (anticancer or anti-inflammatory drugs, nucleic acids, metal based contrast agents) (21-23). High rigidity of the bilayer corresponds to an improved retention of the encapsulated drug, while high microfluidity can lead to a reduction of the incorporation capability (23-25). Modulation of bilayer 
microfluidity can be also achieved through addition of cholesterol, extensively employed in liposomal formulations as stabilizing agent. On the basis of these observations, it appears clear that, when choosing the best formulation for the delivery of a drug, it is of capital importance to find the right compromise between fluidizing components, to maximize encapsulation, and rigidity conferring elements to improve stability and drug retention. Earlier studies reported by our group demonstrated the PNA oligomer PNA-a210 can be encapsulated in liposomes composed of PC/CHO/DSPE-PEG2000 (47/47/6 molar ratio) with an encapsulation efficiency of $1.69 \%$ and that the encapsulation efficiency was improved upon DSPE-PEG post-insertion ( 30\%, EE\%: 2.20). This encapsulation protocol was chosen following results achieved by three different methods: thin film method/sonication, freeze/thawing, and SNALPs (18). Any substantial difference were observed in the encapsulation efficiency achieved by the three procedures. However, liposomes obtained with the freeze/thawing method showed lower polydispersity index with respect to the other preparations. In order to investigate the effect of the lipid bilayer fluidity on the incorporation and retention of PNA-a210, we compared the drug loading capability of liposomal formulations containing lipids with an alkyl chain of eighteen carbon atoms, characterized by different unsaturation degrees of the hydrophobic portion. The effect of the head group was evaluated by studying the encapsulation properties of liposomes prepared with lipids with DOPG. This phospholipid has the same hydrophobic portion of DOPC, but the neutral phosphatidylcholine head group is replaced by the negatively charged phosphatidylglycerol group. Furthermore, each phospholipid was used alone or in combination with an increasing percentage of cholesterol, ranging from 0 to $47 \%$. Stealth liposomes were finally obtained by adding DSPE-PEG2000 (6 mol\%). In order to avoid the shielding effect of PEG during the PNA encapsulation, PEG surface decoration was achieved using the well-known post-insertion method.

\subsection{Preparation of the liposomes}

We investigated the effect of unsaturation of the lipids on the PNA encapsulation capability preparing liposomes composed of two-tailed phospholipids (HSPC, egg PC, DOPC, DOPG) and cholesterol at several molar ratios (10, 20, 30 and $47 \mathrm{~mol}$ (Table 1)). HSPC and DOPC contain zero and two unsaturations respectively (Fig. 1), while egg PC is composed of a mixture of natural phospholipids (average composition in fatty acids as follow: $32.7 \%$ of 16:0, $12.3 \%$ of 18:0, 32.0\% of $18: 1$ and $17.1 \%$ of 18:2), characterized by a highly variable unsaturation degree and hydrophobic chains at sixteen and eighteen carbon atoms. In order to exclude the influence of the encapsulation method on the loading efficiency and to reliably compare the encapsulation efficiency of each liposomal formulation, we applied an unique method for the preparation of all samples. This procedure is based on a combination of thin film/sonication and freeze/thawing methods (Fig. 2). In this new method we reduced the freeze/thawing cycles, because we observed a worsening of some liposomal preparations (in particular DOPG based liposomes) during the freeze/thawing. On the other hand, HSPC based liposomes did not give satisfactory results, in terms of PNA-a210 encapsulation, when freeze/thawing step was not performed. However, due to the different melting temperatures $\left(\mathrm{T}_{\mathrm{m}}\right)$ of the four phospholipids here studied, extrusion of the liposomes was performed at different temperatures $\left(25^{\circ}, 55^{\circ}\right.$ and $\left.65^{\circ} \mathrm{C}\right)$. $\mathrm{T}_{\mathrm{m}}$, also defined as the gel to liquid-crystalline disordered phase transition, represents the temperature required to induce a change in the lipid physical state from the ordered gel phase, where the hydrocarbon chains are fully extended and closely packed, to the disordered liquid crystalline phase, where the hydrocarbon chains are randomly oriented and fluid. The phase transition temperature increases as a function of hydrocarbon chain length, as a consequence of the high packing order favored by van der Waals interactions. The presence of one or more cis double bond into the acyl group destabilizes the interaction between the adjacent chains, thus requiring lower temperatures to prompt the transition 
from solid-gel to liquid-crystalline phase $(26 ; 27)$. Moreover, the addition of cholesterol to a pure phospholipid bilayer causes a broadening of the $\mathrm{T}_{\mathrm{m}}$ of the membrane. According to these considerations, the formulations containing DOPC and DOPG phospholipids, having a negative value of $\mathrm{T}_{\mathrm{m}},-20^{\circ}$ and $-18^{\circ} \mathrm{C}$ respectively, were extruded at room temperature, independently from the cholesterol content. All the liposomes containing HSPC were extruded at $65^{\circ} \mathrm{C}$, well-above their transition temperature $\left(\mathrm{T}_{\mathrm{m}}=55^{\circ} \mathrm{C}\right.$ of the pure phospholipid). Finally, PC based liposomes containing at 0 and $10 \%$ of cholesterol were extruded at room temperature, whereas the others three samples containing higher amounts of cholesterol $(20,30$ and $47 \mathrm{~mol} \%)$ were extruded at $55^{\circ} \mathrm{C}$.

\subsection{Drug loading}

The amount of PNA encapsulated (expressed as $\mu \mathrm{g}$ ), the encapsulation efficiency (EE\%), defined as the weight ratio of encapsulated PNA versus the amphiphilic molecules forming liposomes, and the encapsulation ratio (ER\%), defined as the weight percentage of PNA encapsulated in the liposomes on the total PNA previously added during preparation, are reported in Table 1 . The encapsulated amount of PNA-a210 for each phospholipid is reported in Fig. 3 as a function of the cholesterol percentage. From the inspection of Fig. 3, it clearly appears that the four phospholipids here compared show pronounced difference in terms of encapsulation efficiency. A gradual increase in the encapsulation efficiency occurs at increasing the unsaturation of the alkyl chain, keeping fixed the choline head group (compare results obtained with pure liposomes of HSPC, egg PC and DOPC). Accordingly ER\% moves from 38.2 for pure HSPC liposomes to 62.6 for pure DOPC liposomes. Egg PC and DOPC showed a similar behavior (ER\% 64.5 and 62.6, respectively), being egg PC a mixture of natural phospholipids with a variable unsaturation degree and chain length. On the other hand, a clear increase in the encapsulated amount (ER\% 87.8) was observed for pure DOPG liposomes, as compared to the other formulations. These results indicate that the combination of two unsaturations on the alkyl chain with a negatively charged head group causes a substantial increase in the PNA loading degree. The effect of addition of cholesterol on PNA loading efficiency was evaluated for all the phospholipids selected for this study, using amounts of cholesterol ranging from 10 to $47 \mathrm{~mol} \%$. It is well-known that the insertion of cholesterol can cause an opposite effect on the lipid bilayer fluidity depending on the $\mathrm{T}_{\mathrm{m}}$ value of the neighboring phospholipids (28). Above the $\mathrm{T}_{\mathrm{m}}$, the interaction of rigid cholesterol molecules with hydrocarbon tails of phospholipids causes a stiffening of the lipid bilayer, which becomes less permeable to small water-soluble molecules. On the contrary, a weakening of interactions between the alkyl chains of phospholipids occurs when cholesterol is added at bilayers in their gel state (temperature below the $\left.\mathrm{T}_{\mathrm{m}}\right)$. According to these findings, the addition of low amounts (10 and $\left.20 \mathrm{~mol} \%\right)$ of cholesterol in liposomes composed of the completely saturated HSPC phospholipid, which exhibits a Tm of $55^{\circ} \mathrm{C}$, arouses a weakening of the lipid bilayer. The higher mobility of the bilayer causes an increase of the encapsulation efficiency (ER\% increase from 38 to 48\%), as indicated in Fig. 3 and in Table 1. However, the addition of higher percentages of cholesterol (30 and $47 \mathrm{~mol} \%$ ) does not result in a further increase of the loading capability. On the other hand, the loading capability of liposomes containing DOPC and DOPG, both with two unsaturations and having very low $\mathrm{T}_{\mathrm{m}}$ values, decreases proportionally with increasing the cholesterol content. This trend is more pronounced for DOPC liposomes than for DOPG ones: a decrease of $42 \%$ and $21 \%$ of the encapsulation ratio was observed for DOPC-47 and DOPG-47, respectively. This different trend beyond validates the hypothesis that also the head group of the phospholipid contributes at modifying the loading capability of liposomes. Due to the high complexity of natural egg PC composition, it is not easy to focus and describe the influence of the cholesterol on its loading properties.

\subsection{Structural characterization}

After preparation, mean diameters, polydispersity indexes (P.I.) and Z potentials $(\zeta)$ of empty liposomes and PNA loaded liposomes, before and after post-insertion of DSPE-PEG2000, were 
assessed using Dynamic Light Scattering (DLS) technique (see Table 2, S1, S2 and S3). The size of all the empty liposomes is in good agreement with the literature and is not significantly affected by the cholesterol percentage used in the formulation (Table 2). All the formulations showed a slight reduction of the liposomal dimensions after the loading of PNA-a210, independently from the cholesterol percentage. The variation of the mean diameter of PNA loaded liposomes as a function of the cholesterol percentage before and after post-insertion of DSPE-PEG2000, is reported in Fig. 4. After post insertion of the DSPE-PEG2000, DLS intensity profiles of DOPC and DOPG show a moderate increase of the mean diameter ranging between 5 and $10 \%$ of the size before postinsertion. On the contrary, the post-insertion of DSPE-PEG2000 in egg PC based liposomes causes a decrease of the liposome size. This decrease probably depends from the insertion of a phospholipid with two saturated C-18 chains in a bilayer of saturated and unsaturated lipids at C-16 and $\mathrm{C}-18$ chains, which produces a condensing and ordering effect on the acyl chains, resulting in the contraction of the liposomal size. Z potential values of all the PNA filled liposomes are also reported in Tables 2, S1, S2 and S3. Both liposomes containing phosphatidylcholine and phosphatidylglycerol as head groups are negatively charged. As expected, liposomes formulated using the negative DOPG lipid have a more negative $\zeta$ value $(-48<\zeta<-61 \mathrm{mV})$. Z potential for PNA-a210 in water solution was also measured in order to evaluate the occurrence of electrostatic interactions between the negative liposomal surface and PNA-a210. $\zeta$ value of PNA was found close to zero (data not shown), thus suggesting that any electrostatic interaction occurs. After DSPE-PEG2000 post-insertion, all the liposomes have $\zeta$ values ranging from - 18 to $-33 \mathrm{mV}$, thus indicating that polyethylene glycol shields the charge of the phospholipids.

Stability of PNA loaded liposomes was evaluated in vitro in fetal bovine serum. Liposomes were incubated with serum and the evolution of their dimensions was checked by DLS measurements at different time points up to $72 \mathrm{~h}$. It is well-known that both liposome composition and charge can affect the stability of liposomes in serum (29). Negatively charged liposomes containing only one phospholipid show low stability in serum; the insertion of cholesterol results in the improvement of liposome stabilitydue to membrane modification and consequent inhibition of the interactions between liposomes and serum proteins. Liposomes here prepared do not show signification variations of their size upon the time (data not shown), thus indicating a good stability. A similar result was previously observed by us with liposomes composed of egg PC/Chol/DSPE-PEG2000 at 47/47/6 molar ratio (18) In our opinion this result can be explained on the basis of the similitude between the external surface of the liposomes here prepared with the post-insertion of DSPEPEG2000.

\subsection{PNA-a210 release}

On the basis of the drug loading data, only DOPG based liposomes were selected to be further investigated for the release of PNA from liposomes. The release profile of PNA-a210 from DOPG based liposomes prepared at different amounts of cholesterol (0, 10, 20, 30 and $47 \mathrm{~mol} \%)$ was studied within $72 \mathrm{~h}$, using a dialysis membrane immersed in phosphate buffer at $37^{\circ} \mathrm{C}$. As expected, the percentage of PNA released from the liposomes is strongly influenced by the amount of cholesterol of each formulation (Fig. 5). From the comparison of the profiles reported in Fig. 5, the highest release percentage (55\%) was observed for the liposomal formulation which lacks cholesterol. The addition of a low (10\%) amount of cholesterol causes a better retention of the PNA in the inner liposomal compartment and a decrease of release percentage $(\sim 34 \%)$. This effect is more evident at $20 \mathrm{~mol} \%(\sim 27 \%)$, whereas the further increase of cholesterol does not produce significant variations. On the contrary, a decrease of the release was observed when the cholesterol percentage is brought at $47 \mathrm{~mol} \%$. This behavior is expected for the release of hydrophilic molecules encapsulated in liposomal formulations containing cholesterol quantity above $40 \%$ (30). In accordance with drug release results, the best percentage of cholesterol to use should be ranged between 20 and $30 \%$. 


\section{Conclusions}

PNA loading capability of liposomes composed of saturated (HSPC) and unsaturated (Egg PC, DOPC and DOPG) commercial phospholipids was evaluated in terms of encapsulation efficiency and of encapsulation ratio. The effect of increasing amounts of cholesterol in the liposomal composition was also investigated. Results indicate that the PNA loading is strongly affected by both unsaturation of the lipid alkyl chains and by the charge of the head group. A gradual increase in the encapsulation efficiency occurs at increasing the unsaturation of the alkyl chain, keeping fixed the choline head group. The replacement of choline head group with the negatively charged glycerol causes a further increase in the PNA loading degree. The insertion of low amount of cholesterol (10-20\%) in DOPG based liposomes provides a slight decrease of the PNA loading. In addition, an amount of cholesterol ranging between 20 and 30\% causes a slowing down of the PNA's release from the liposomal formulation. For their encapsulation and the release properties, we can conclude that PEGylated DOPG liposomes with a percentage of cholesterol of 10-20\% can be considered the best formulation for the loading of PNA-a210. Future investigations will be carried out to understand whether the composition of the PNA oligomers (i.e. the purine:pyrimidine ratio) affects the loading capability of DOPG based liposomes.

\section{Acknowledgements}

We are indebted with the Italian Minister for Research (M.I.U.R.) for financial support under FIRB 'RENAME' RBAP114AMK project. The authors thank AIRC for financial support (AIRC grant IG2014\#15764).

Appendix A. Supplementary data

Supplementary data associated with this article can be found, in the online version.

\section{References}

[1] C. Avitabile, A. Cimmino, A. Romanelli, Journal of medicinal chemistry 57 (2014) 10220-40.

[2] P.E. Nielsen, M. Egholm, R.H. Berg, O. Buchardt, Science 254 (1991) 1497-500.

[3] V.V. Demidov, V.N. Potaman, M.D. Frank-Kamenetskii, M. Egholm, O. Buchard, S.H. Sonnichsen, P.E. Nielsen, Biochemical pharmacology 48 (1994) 1310-3.

[4] R. Tonelli, et al., Molecular cancer therapeutics 4 (2005) 779-86.

[5] Y.M. Bae, M.H. Kim, G.S. Yu, B.H. Um, H.K. Park, H.I. Lee, K.T. Lee, Y.D. Suh, J.S. Choi, Journal of controlled release : official journal of the Controlled Release Society 175 (2014) 54-62.

[6] A.G. Torres, M.M. Fabani, E. Vigorito, D. Williams, N. Al-Obaidi, F. Wojciechowski, R.H. Hudson, O. Seitz, M.J. Gait, Nucleic acids research 40 (2012) 2152-67.

[7] C. Avitabile, M. Saviano, L. D'Andrea, N. Bianchi, E. Fabbri, E. Brognara, R. Gambari, A. Romanelli, Artificial DNA, PNA \& XNA 3 (2012) 88-96.

[8] A. Gupta, R. Bahal, M. Gupta, P.M. Glazer, W.M. Saltzman, Journal of controlled release : official journal of the Controlled Release Society (2016).

[9] H.V. Jain, D. Verthelyi, S.L. Beaucage, Rsc Advances 5 (2015) 65245-65254.

[10] H. Fang, K. Zhang, G. Shen, K.L. Wooley, J.S. Taylor, Molecular pharmaceutics 6 (2009) 615-26.

[11] P.R. Berthold, T. Shiraishi, P.E. Nielsen, Bioconjugate chemistry 21 (2010) 1933-1938.

[12] T. Shiraishi, P.E. Nielsen, Bioconjugate chemistry 23 (2012) 196-202.

[13] T. Shiraishi, P.E. Nielsen, Methods in molecular biology 751 (2011) 209-21.

[14] S. Pensato, M. Saviano, A. Romanelli, Expert opinion on biological therapy 7 (2007) 1219-32.

[15] C. Avitabile, L. Moggio, G. Malgieri, D. Capasso, S. Di Gaetano, M. Saviano, C. Pedone, A. Romanelli, PloS one 7 (2012) e35774. 
[16] J. Valero, T. Shiraishi, J. de Mendoza, P.E. Nielsen, Chembiochem : a European journal of chemical biology 16 (2015) 1593-600.

[17] N.A. McNeer, J.Y. Chin, E.B. Schleifman, R.J. Fields, P.M. Glazer, W.M. Saltzman, Molecular therapy : the journal of the American Society of Gene Therapy 19 (2011) 172-80.

[18] C. Avitabile, A. Accardo, P. Ringhieri, G. Morelli, M. Saviano, G. Montagner, E. Fabbri, E. Gallerani, R. Gambari, A. Romanelli, Bioconjugate chemistry 26 (2015) 1533-41.

[19] N. Bianchi, C. Zuccato, I. Lampronti, M. Borgatti, R. Gambari, BMB reports 42 (2009) 493-9.

[20] E. Ducat, M. Brion, F. Lecomte, B. Evrard, G. Piel, AAPS PharmSciTech 11 (2010) 966-75.

[21] A.M. Saetern, G.E. Flaten, M. Brandl, AAPS PharmSciTech 5 (2004) e40.

[22] P. Ringhieri, R. lannitti, C. Nardon, R. Palumbo, D. Fregona, G. Morelli, A. Accardo, International journal of pharmaceutics 473 (2014) 194-202.

[23] B. Selvi, S. Patel, M. Savva, Journal of pharmaceutical sciences 97 (2008) 4379-90.

[24] S. Kruszewski, D.M. Kruszewska, Acta Physica Polonica A 118 (2010) 99-102.

[25] C.D. Modi, R.S.R. Murthy, Der Pharmacia Lettre 3 (2011) 34-47.

[26] W.C. Hung, M.T. Lee, F.Y. Chen, H.W. Huang, Biophysical journal 92 (2007) 3960-7.

[27] T. Rog, M. Pasenkiewicz-Gierula, I. Vattulainen, M. Karttunen, Biochimica et biophysica acta 1788 (2009) 97-121.

[28] F. Ercole, M.R. Whittaker, J.F. Quinn, T.P. Davis, Biomacromolecules 16 (2015) 1886-914.

[29] T. Hernandez-Caselles, J. Villalain, J.C. Gomez-Fernandez, Molecular and cellular biochemistry 120 (1993) 119-26.

[30] M.L. Briuglia, C. Rotella, A. McFarlane, D.A. Lamprou, Drug delivery and translational research 5 (2015) 231-42. 


\section{Figures}

A)

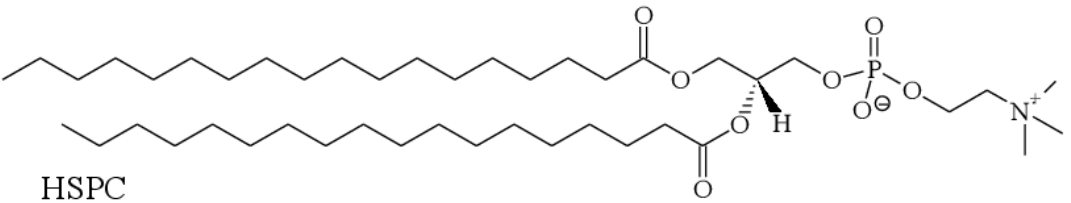

B)

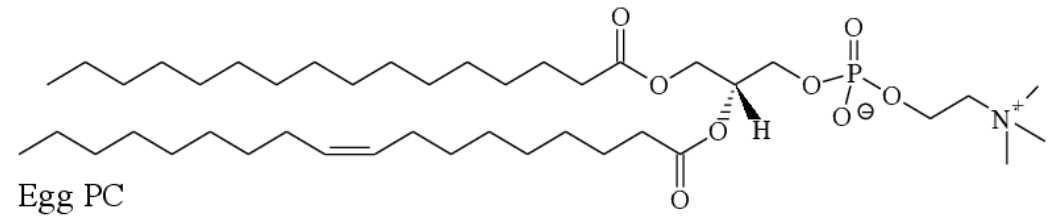

C)

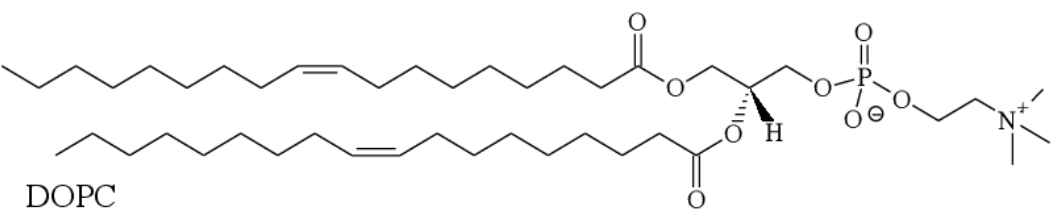

D)

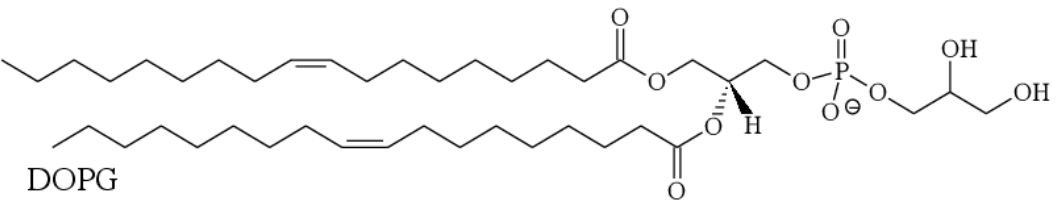

E)

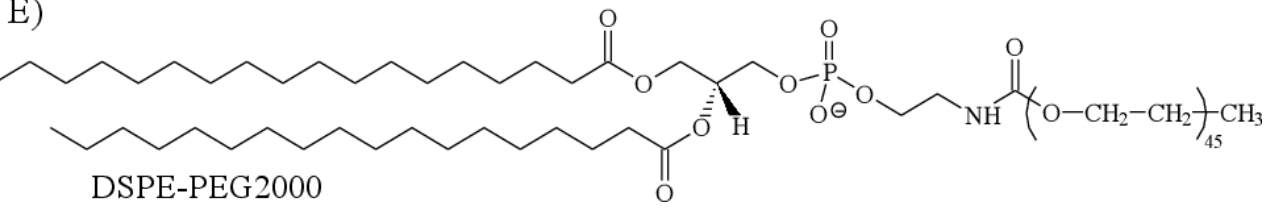

Fig.1. Schematic representation of phospholipids used for the liposomal formulations: HSPC, egg PC, DOPC, DOPG and DSPE-PEG2000. For egg PC, which is a mixture of natural phospholipids, is here only represented the predominant fatty acid. 


\section{Thin film/sonication method: empty liposome preparation}

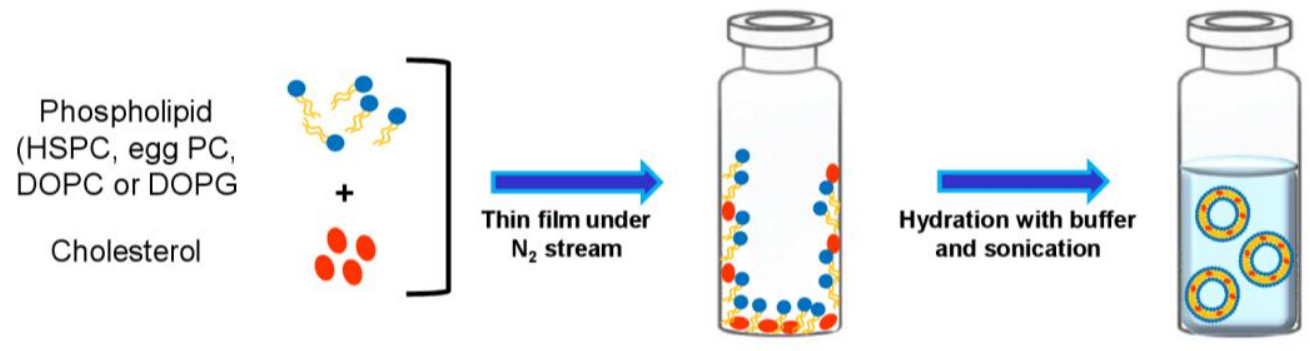

\section{Freeze/Thawing method: PNA encapsulation}

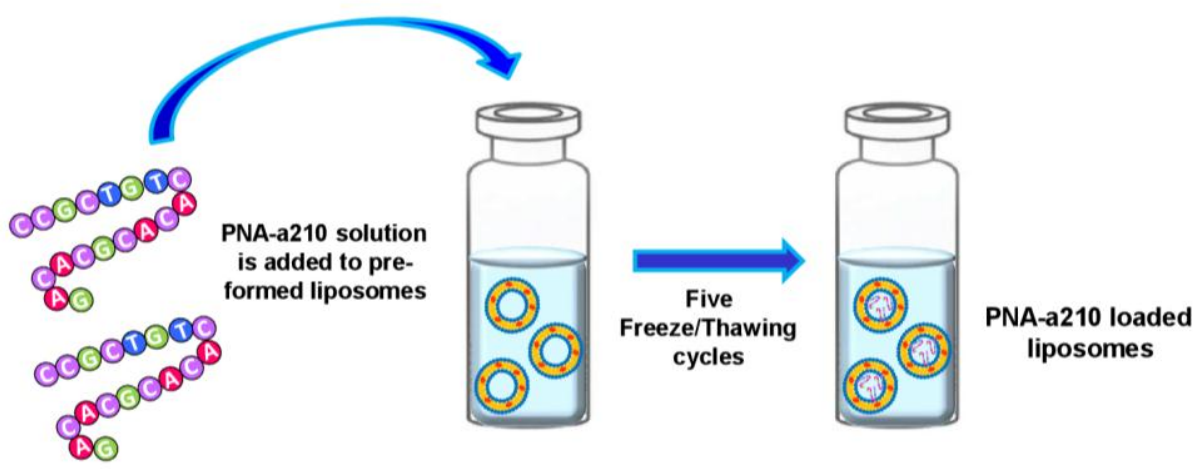

\section{Post-insertion of DSPE-PEG2000}

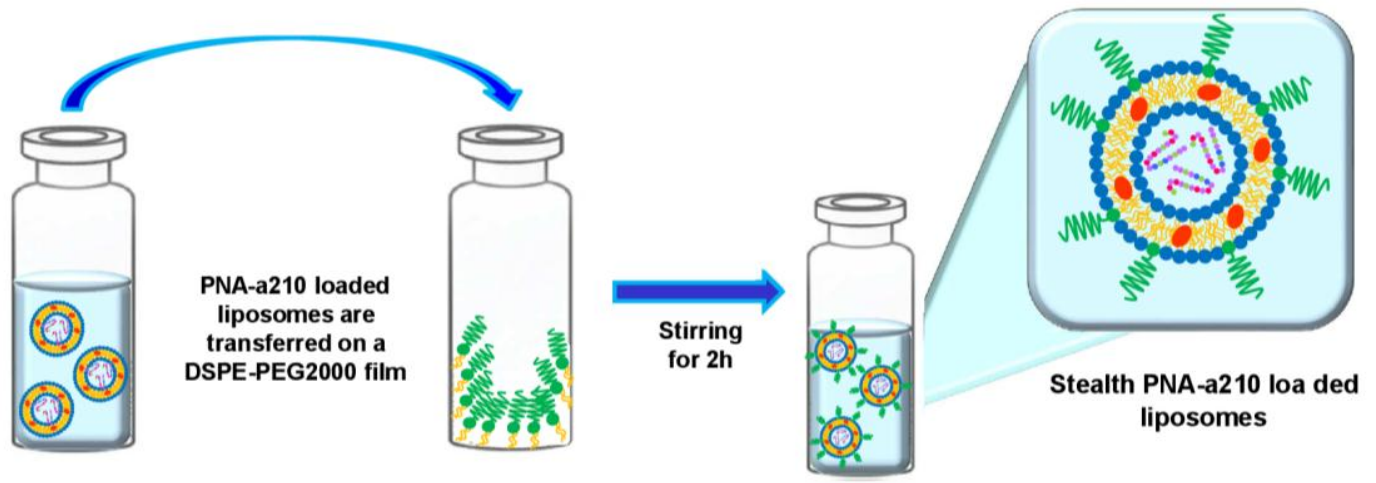

Fig.2. Preparation of PNA loaded liposomes in three steps: 1) the mixture of lipids was dried under vacuum to form a lipid film on the wall of the vial, then the film was hydrated and sonicated with the buffer; 2) PNA solution is added to the vial and liposomes undergone to five freeze/thawing cycles; 3) liposomes were made stealth by adding DSPE-PEG2000 using the post-insertion method. 


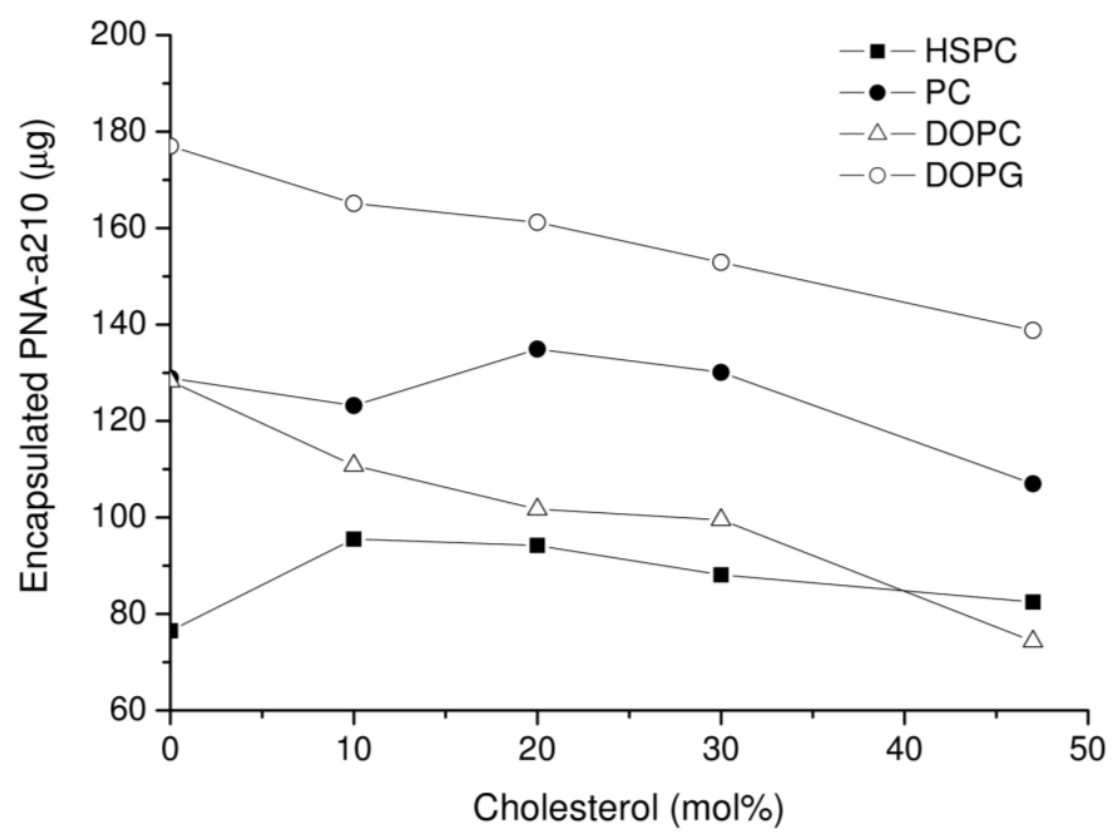

Fig.3. Amount of PNA-a210 ( $\mu \mathrm{g})$ encapsulated in $10 \mathrm{mM}$ liposomes composed of HSPC, egg PC, DOPC or DOPG and cholesterol at different percentages. $\mu \mathrm{g}$ of PNA-a210 encapsulated in liposomes are reported as the mean of the three experiments. 

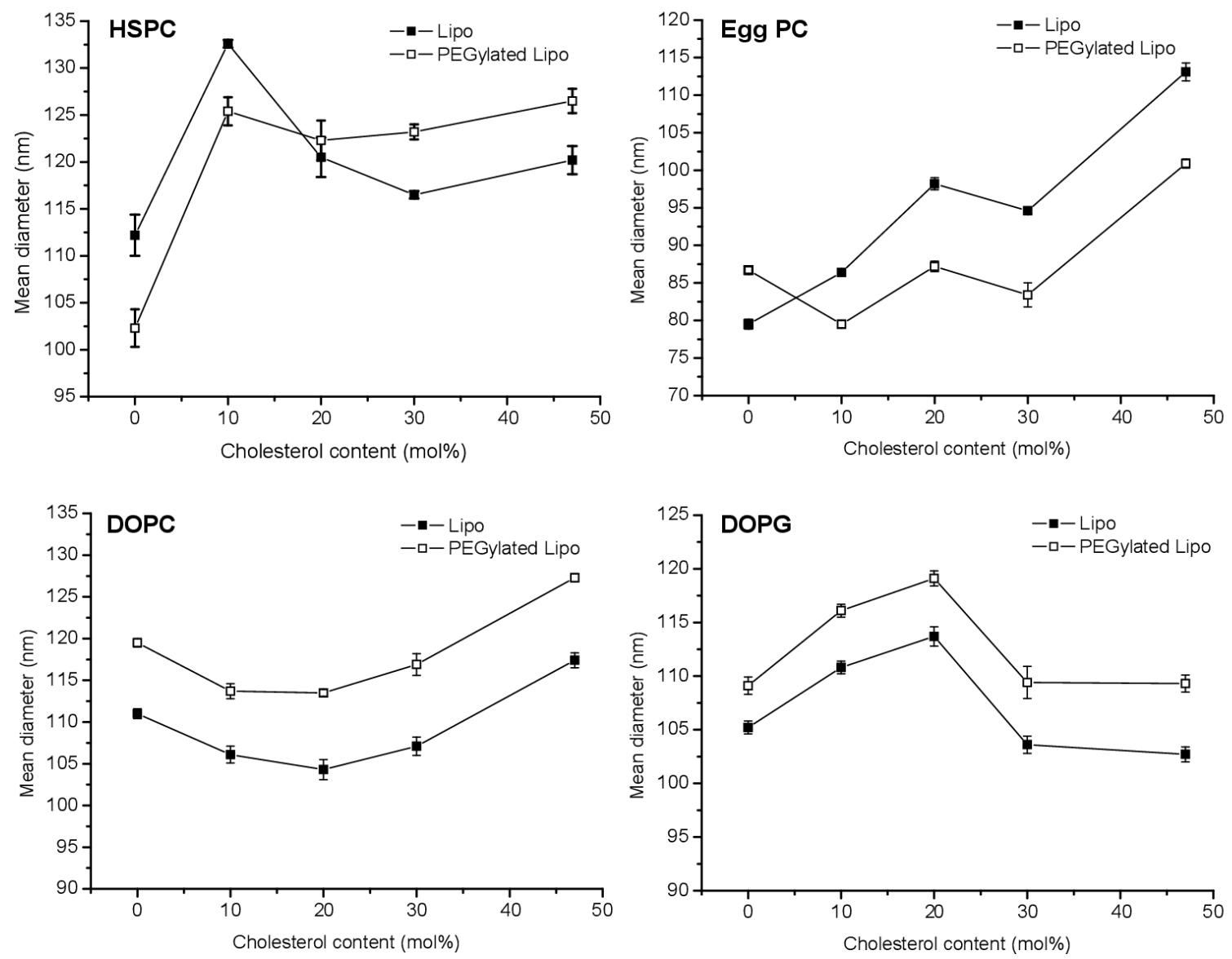

Fig.4. Mean diameter of all the PNA loaded liposomes as a function of the cholesterol content (mol\%) before (Lipo) and after post-insertion (PEGylated-Lipo) of DSPE-PEG2000. Diameter values are reported with the error bars as the mean of the three experiments. 


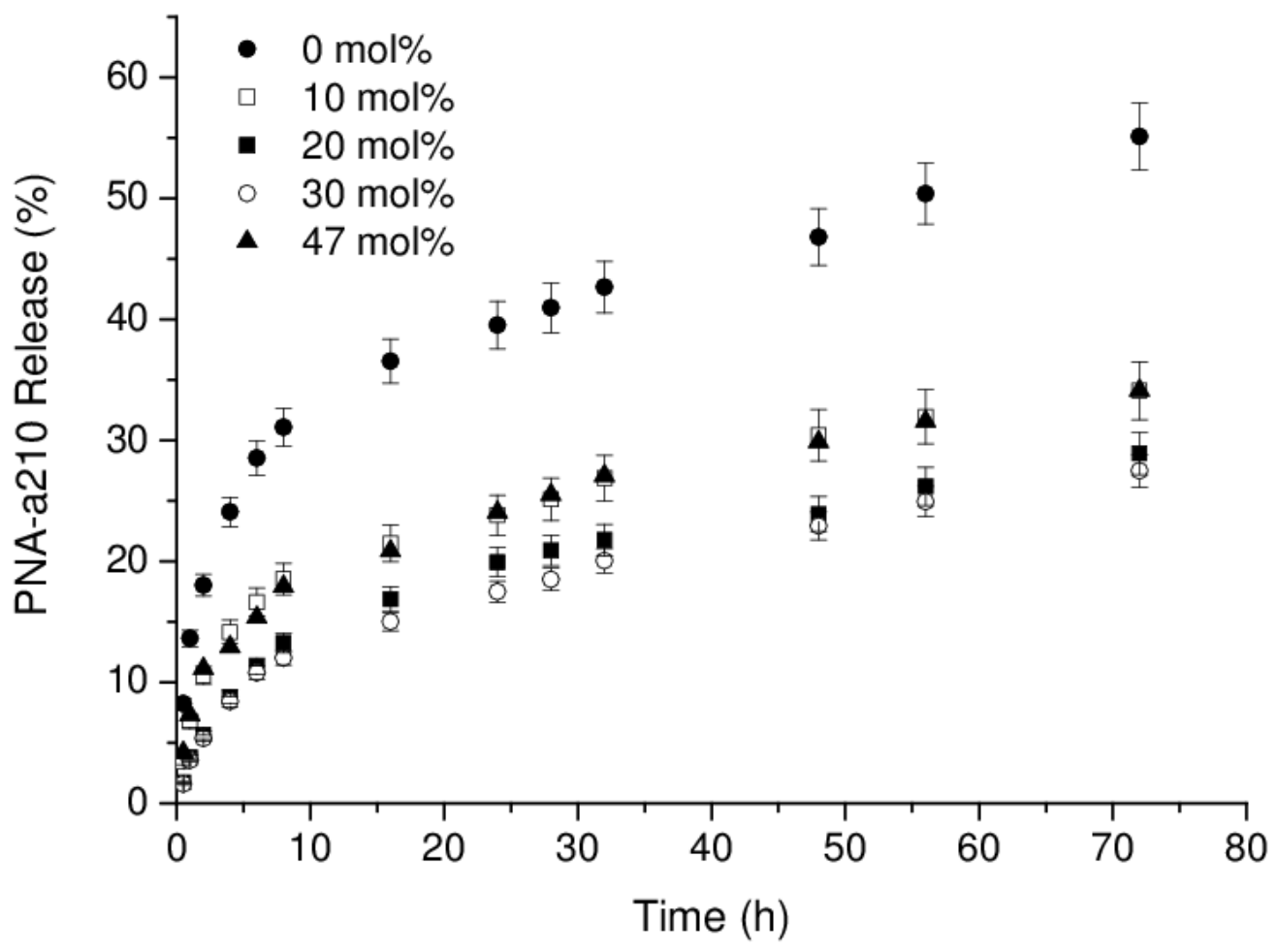

Fig.5. Release of PNA-a210 by DOPG based liposomes prepared at different percentages of cholesterol $(0,10,20,30$ and $47 \mathrm{~mol} \%)$. The amount of PNA-a210 released (\%) was estimated by $\mathrm{UV}-\mathrm{V}$ is at $260 \mathrm{~nm}$ and reported with the error bars as function of the time in hours. 
Table 1: Liposomal formulations and $\mu \mathrm{g}$ of PNA-a210 encapsulated in $1 \mathrm{~mL}$ of liposomes at 10 $\mathrm{mM}$ lipid concentration. EE\% and ER\% for each sample are also reported.

\begin{tabular}{|c|c|c|c|c|c|}
\hline & Sample & $\begin{array}{c}\text { Cholesterol } \\
\text { amount (mol) }\end{array}$ & PNA $(\mu g)$ enc & ER\% & EE\% \\
\hline \multirow{5}{*}{ 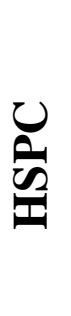 } & HSPC-94 & ---- & 76.5 & 38.2 & 1.67 \\
\hline & HSPC-84 & 10 & 95.5 & 47.8 & 2.34 \\
\hline & HSPC-74 & 20 & 94.2 & 47.1 & 2.45 \\
\hline & HSPC-64 & 30 & 88.1 & 44.1 & 2.43 \\
\hline & HSPC-47 & 47 & 82.5 & 41.1 & 2.53 \\
\hline \multirow{5}{*}{$\bigcup_{1}$} & PC-94 & ---- & 128.9 & 64.5 & 2.79 \\
\hline & PC-84 & 10 & 123.2 & 61.6 & 2.98 \\
\hline & PC-74 & 20 & 134.9 & 67.5 & 3.49 \\
\hline & PC-64 & 30 & 130.1 & 65.0 & 3.55 \\
\hline & PC-47 & 47 & 107.0 & 53.5 & 3.27 \\
\hline \multirow{5}{*}{$\begin{array}{l}\varrho \\
0 \\
0\end{array}$} & DOPC-94 & ---- & 128.3 & 62.6 & 2.72 \\
\hline & DOPC-84 & 10 & 110.8 & 54.0 & 2.64 \\
\hline & DOPC-74 & 20 & 101.7 & 49.6 & 2.57 \\
\hline & DOPC-64 & 30 & 99.52 & 48.5 & 2.69 \\
\hline & DOPC-47 & 47 & 74.33 & 36.3 & 2.23 \\
\hline \multirow{5}{*}{$\begin{array}{l}0 \\
0 \\
0\end{array}$} & DOPG-94 & ----- & 177.0 & 87.8 & 3.66 \\
\hline & DOPG-84 & 10 & 165.1 & 82.6 & 3.88 \\
\hline & DOPG-74 & 20 & 161.2 & 80.6 & 4.03 \\
\hline & DOPG-64 & 30 & 152.9 & 76.4 & 4.07 \\
\hline & DOPG-47 & 47 & 138.8 & 69.4 & 4.14 \\
\hline
\end{tabular}


Table 2: Structural parameters (mean diameter, P.I., $D, \zeta$ ) from DLS measurements for DOPG based liposomes empty or encapsulating PNA-a210, before and after DSPE-PEG2000 postinsertion. Standard deviation (SD) for values of diameter, diffusion coefficient and $\mathrm{Z}$ potential are also reported.

\begin{tabular}{lcccccccc}
\hline \multirow{2}{*}{ Sample } & \multicolumn{2}{c}{$\begin{array}{c}\text { Mean diameter } \pm \text { SD } \\
(\mathbf{n m})\end{array}$} & \multicolumn{2}{c}{ P.I. } & \multicolumn{2}{c}{$\mathbf{D}\left[\mathbf{m}^{2} \mathbf{s}^{-1}\right] \mathbf{x} 1 \mathbf{0}^{-12}$} & \multicolumn{2}{c}{$\zeta \mathbf{m V} \pm$ SD } \\
& \multicolumn{1}{c}{ Empty } & PNA & Empty & PNA & Empty & PNA & Empty & PNA \\
\hline DOPG-94 & $118 \pm 46$ & $106 \pm 44$ & 0.107 & 0.112 & $4.2 \pm 1.6$ & $4.7 \pm 1.9$ & $-27.3 \pm 1.7$ & $-51.3 \pm 1.7$ \\
PEGylated & $124 \pm 54$ & $110 \pm 41$ & 0.122 & 0.102 & $4.0 \pm 1.7$ & $4.5 \pm 1.7$ & $-18.2 \pm 0.5$ & $-27.7 \pm 3.1$ \\
DOPG-94 & & & & & & & & \\
DOPG-84 & $112 \pm 44$ & $111 \pm 56$ & 0.093 & 0.155 & $4.4 \pm 1.7$ & $4.4 \pm 2.2$ & $-26.6 \pm 3.4$ & $-61.0 \pm 1.1$ \\
PEGylated & $116 \pm 40$ & $117 \pm 68$ & 0.086 & 0.193 & $4.3 \pm 1.5$ & $4.2 \pm 2.4$ & $-18.7 \pm 0.4$ & $-31.3 \pm 0.7$ \\
DOPG-84 & & & & & & & & \\
DOPG-74 & $116 \pm 37$ & $114 \pm 50$ & 0.072 & 0.136 & $4.3 \pm 1.4$ & $4.3 \pm 1.9$ & $-37.2 \pm 2.3$ & $-58.7 \pm 2.1$ \\
PEGylated & $121 \pm 34$ & $119 \pm 51$ & 0.042 & 0.132 & $4.1 \pm 1.1$ & $4.1 \pm 1.8$ & $-24.5 \pm 1.5$ & $-33.3 \pm 1.5$ \\
DOPG-74 & & & & & & & & \\
DOPG-64 & $117 \pm 41$ & $104 \pm 53$ & 0.079 & 0.165 & $4.2 \pm 1.5$ & $4.7 \pm 2.4$ & $-37.8 \pm 1.9$ & $-54.7 \pm 1.4$ \\
PEGylated & $118 \pm 46$ & $108 \pm 50$ & 0.091 & 0.134 & $4.2 \pm 1.6$ & $4.6 \pm 2.1$ & $-33.0 \pm 0.9$ & $-32.2 \pm 0.5$ \\
DOPG-64 & & & & & & & & \\
DOPG-47 & $112 \pm 42$ & $103 \pm 57$ & 0.092 & 0.202 & $4.4 \pm 1.6$ & $4.8 \pm 2.6$ & $-31.0 \pm 0.3$ & $-48.6 \pm 1.1$ \\
PEGylated & $115 \pm 45$ & $109 \pm 55$ & 0.106 & 0.154 & $4.3 \pm 1.7$ & $4.5 \pm 2.3$ & $-35.0 \pm 3.3$ & $-31.3 \pm 0.3$ \\
DOPG-47 & & & & & & & & \\
\hline
\end{tabular}

